\title{
Impaired Executive Function in Everyday Life: A Predictor of OCD Relapse?
}

\author{
Nathalie Fournet ${ }^{1 *}$, Ouafae Achachi' ${ }^{1}$ Arnaud Roy ${ }^{2,3}$, Jérémy Besnard ${ }^{2}$, Céline Lancelot ${ }^{2}$, \\ Didier Le Gall'2, Martine Bouvard1 \\ ${ }^{1}$ Université Grenoble Alpes, Université Savoie Mont Blanc, CNRS, LPNC, Grenoble, France \\ ${ }^{2}$ Laboratoryof Psychology des Pays de la Loire, EA4638, Université Bretagne Loire-Université d’Angers, Angers, France \\ ${ }^{3}$ Reference Center of Learning Disorders-Centre Référent des Troubles d'Apprentissage, Centre de Compétence Nantais de \\ Neurofibromatose, Hôpital Femme-Enfant-Adolescent, CHU de Nantes, Nantes, France
}

Email: *nathalie.fournet@univ-smb.fr

How to cite this paper: Fournet, N., Achachi, O., Roy, A., Besnard, J., Lancelot, C., Le Gall, D. and Bouvard, M. (2019) Impaired Executive Function in Everyday Life: A Predictor of OCD Relapse? Journal of Behavioral and Brain Science, 9, 90-107. https://doi.org/10.4236/jbbs.2019.93008

Received: December 7, 2018

Accepted: March 2, 2019

Published: March 5, 2019

Copyright (C) 2019 by author(s) and Scientific Research Publishing Inc. This work is licensed under the Creative Commons Attribution International License (CC BY 4.0).

http://creativecommons.org/licenses/by/4.0/

\begin{abstract}
Objectives: In the present study, we set out to establish whether executive function in everyday life is impaired in obsessive-compulsive disorder patients, and if it is more impaired (or not) in patients who relapsed after cognitive behavioural therapy than in treatment-naïve obsessive-compulsive disorder patients. Method: The Behavior Rating Inventory of Executive FunctionAdult Version (BRIEF-A) was used to measure executive function in everyday life in three groups of 19 participants: treatment-naïve obsessive-compulsive disorderpatients, obsessive-compulsive disorder patients having relapsed after CBT, and healthy controls. Results: The BRIEF-A results revealed an impairment in executive function in the treatment-naïve and relapsed obsessive-compulsive disorder groups, relative to the healthy control group. There was no significant difference in executive function between the two groups of patients. Conclusions: These results show that impaired executive function is not associated with relapse in patients with obsessive-compulsive disorder having undergone cognitive behavioural therapy.
\end{abstract}

\section{Keywords}

Obsessive Compulsive Disorder, Relapse, Executive Function, Cognitive Behavioural Therapy

\section{Introduction}

Obsessive-compulsive disorder (OCD) is a neuropsychiatric illness characterized by persistent, intrusive thoughts (obsessions) generally accompanied by ritualized/repetitive acts or thoughts (compulsions). This disorder can often severely 
impair a person's ability to function in everyday life. Neuropsychological studies have attempted to understand the cognitive processes underlying OCD. Chamberlain, Blackwell, Fineberg, Robbins and Sahakain [1] suggested to conceptualize OCD as a "lateral orbitofrontal loop dysfunction" leading to impairments in tasks requiring executive function (mainly inhibitory control and mental flexibility).

Executive function (EF) encompasses a set of interrelated (although possibly distinct) abilities for guiding and monitoring cognitions, behaviour and emotions; they notably include set shifting, inhibition, working memory, and planning [2] [3]. An impairment in EF will necessarily affect a person's behaviour in everyday life, by limiting his/her ability to adjust to environmental demands or changes. In neuropsychological studies, impairments in EF have been linked to damage to or dysfunction of the prefrontal cortex and the disruption of frontosubcortical pathways [4] [5]. Neuroimaging studies have highlighted a link between OCD and the brain areas involved in EF-mainly the prefrontal cortex and the cortico-striato-thalamo-cortical circuits [6] [7] [8]. These sets of converging evidence have prompted researchers to examine OCD from a neurobiological perspective.

Impairments in EF are frequently observed in OCD (see [9] for a review). But if $\mathrm{EF}$ impairments have been linked to prefrontal and frontosubcortical dysfunctions in $\mathrm{OCD}$, the specific nature and pattern of EF impairments remain unclear: is it general or is there specific impairments on more specific EF components? In a group of patients with OCD and in a control group of patients with anxiety disorder, Bannon et al. [10] studied inhibition, set shifting, planning, verbal fluency and working memory; they found that only some aspects of EF (set shifting and inhibition) are specifically impaired in OCD. Olley, Malhi and Sachdev [11] reviewed memory and EF in OCD. They evidenced a neuropsychological profile in OCD with a primary impairment in EF. The memory impairment in OCD seemed to result from a strategic deficit in organization during encoding (see also [12]). In a meta-analysis, Snyder et al. [9] concluded that OCD patients showed EF deficits in tasks measuring inhibition (e.g. the Stroop test and the stop signal task), shifting (e.g. the Wisconsin Card Sorting Test), working memory and updating (e.g. the digitspan backward, Corsi block span and n-back tasks), and planning. These findings suggest that OCD is associated with broad impairments in EF and not just the selective impairments in shifting or inhibition hypothesized by Bannon et al. [10]. However, the nature of EF impairment in patients with OCD had not been unambiguously demonstrated as the abilities of OCD patients to shift set, organize, plan, and quickly solve problems have yielded contradictory results [13]; those contradictory results might reflect the impact of psychotropic medication, taken by some participants, on cognition; the effect of OC symptoms severity on cognitive performance; the potential impact of educational level on neuropsychological functioning; the effect of possible comorbidities (such as depression and anxiety); specific OCD symptom presentations (e.g., checking, contamination/cleaning or sexual/religious obsessions) 
or methodological differences between studies (i.e. type of tests used to evaluate a specific function, presence or absence of a control group...), [13]. Abramowitch, Abramowitz and Mittelman [14] performed a meta-analysis of 115 studies on the neuropsychology of OCD. Medium-sized effects were found for all executive dimensions (planning, response inhibition and set-shifting), with poorer overall performance in OCD. Statistically significant effect sizes were found across various cognitive domains, including executive function, more specifically, planning, response inhibition, set shifting, indicating reduced performance in OCD patients compared to healthy controls. However, the extent of neuropsychological impairment across specific EF varied. The Stroop test gave divergent findings for response inhibition (usually seen as an endophenotypic marker for OCD), with medium weighted mean effect size found for the Stroop interference score and commission errors, whereas small effect sizes were found for working memory. It also appears that certain cognitive tasks (e.g. the Wisconsin Card Sorting Test) are not appropriate for psychopathological testing and are not sufficiently specific for a specific function (e.g. set-shifting).

Hence, executive functions display both unity and diversity, and the available tasks are likely to measure more than one specific executive function. EFs are usually assessed using performance-based, standardized neuropsychological tests. However, their ecological validity has been questioned, because there is little correspondence between the cognitive resources tapped in the test condition (artificial, more structured settings), and those tapped in more natural, "real-world" ones [15]. Efforts have thus been made to develop tests with higher ecological validity by means of self and informant questionnaires to rate the patient's everyday behavior, in various contexts (home, work...). McNamara et al. [16] used the Behavioral Rating Inventory of Executive Function (BRIEF) to measure several domains of EF in everyday life in paediatric OCD patients at several time points during a course of treatment. The ecological validity of many performance-based EF measures has been questioned in the literature [17]. Given the need to evaluate multiple executive domains, a variety of questionnaires (such as the BRIEF) have been used to investigate the everyday behavioural manifestations of EF (i.e. emotional, behavioural, and metacognitive skills). McNamara et al. [16] found that impairments in shifting, inhibition, emotional control, planning/organizing, monitoring, and initiating domains of EF were predictive of higher mean obsessive compulsive severity scores throughout the treatment and a worse response to treatment.

Cognitive-behavioral therapy (CBT), particularly exposure and response prevention, is one of the first line treatment for OCD, and consistent results show neurobiological changes as a result of therapy, with normalizations of neurofunctional dysregulations in key regions of the fronto-striatal network (mainly orbitofrontal cortex, nucleus accumbens, caudate nucleus and ventrolateral prefrontal cortex) [18] [19]. These structures also play a crucial role for EF. One could thus expect a reduction of EF dysfunction after CBT. Voderholzer et al. 
[20] showed that after CBT, OCD patients had significantly improved test results (relative to controls) in various cognitive domains, including verbal fluency (measuring the EF of initiation) and set shifting ability. The researchers suggested that impaired cognitive functions in OCD are partly state-dependent and thus reversible after treatment. It is not clear why some OCD patients are refractory to treatment with medication (mainly antidepressants) and/or CBT (primarily exposure and response prevention) or why some patients relapse after treatment. D'Alcante et al. [21] found that OCD patients with better cognitive and executive abilities at baseline (mainly mental flexibility and inhibition) were more likely to respond to either CBT or fluoxetine. On the other hand, it has been suggested that impairments in inhibition and mental flexibility are involved in post-treatment relapse. Bannon et al. [10] referred to these deficits in inhibition and mental flexibility as trait markers of OCD that might reflect the short-term effects of psychotherapy on cognitive processes. Over the course of the disorder, these deficits might remain stable or might become more or less intense: "the persistence of [executive] underlying deficits may be responsible for, or be associated with mechanisms underpinning further relapses" (Bannon et al. [10], p. 10). The status of executive abilities after OCD relapse therefore raises questions. A relapse is defined as a return to the same level of symptoms as before treatment. If EF dysfunction in OCD is trait-dependent, it may be responsible for further relapses and relapsed patients could exhibit greater executive deficits than treatment-naïve patients or patients who will respond to the CBT. On the other hand, if impaired EF in OCD is reversible after treatment (thus state-dependent, or partly), when OCD patients relapse after treatment, they could again exhibit EF deficits. The return to the same level of obsessive-compulsive symptoms could be linked to a return to the same level of EF impairment after relapse than before treatment.

Given the EF impairments observed in OCD, we hypothesized that:

1) Relative to a group of healthy control participants, a group of OCD patients having relapsed after CBT (the relapsed OCD group) and a group of symptomatic OCD patients never having undergone CBT (the treatment-naïve OCD group) would display a greater impairments in EF in everyday life (as measured by the Behavioral Rating Inventory of Executive Function-Adult Version, BRIEF-A) in general and in inhibition and flexibility in particular. If the two groups of OCD patients exhibit executive deficits in the BRIEF-A, are those deficits similar as they are both experiencing OCD symptoms, or is the relapsed group more impaired?

2) Higher impairments in EF could be predictive of higher obsessive - compulsive severity scores.

\section{Material and Methods}

\subsection{Participants}

Three groups of 19 participants were included in the study and assessed during a 
period of 1 year: treatment-naïve OCD patients (11 males and 8 females), OCD-relapse patients (12 males and seven females) and 19 healthy control participants (10 males and nine females). The groups were matched with regard to age, gender and educational level (Table 1).

The patients were recruited from two different healthcare establishments: Relapsed OCD patients were recruited from the inpatient unit of a psychiatric hospital and treatment-naïve OCD patients were from private practice. Healthy control participants were recruited by advertisements in our University Consulting Unit and elsewhere, and were selected to match the patient groups for age, gender ratio and educational level.

All the patients had a clinical diagnosis of OCD, according to the DSM-IV criteria, following a semi-structured Mini International Neuropsychiatric Interview (MINI) with trained psychologists [22]. Trained clinicians had subsequently checked that the patients also met the DSM-5 criteria (American Psychiatric Association, [23]) for OCD.

The treatment-naïve OCD group comprised patients newly admitted for a course of CBT and who had never undergone psychotherapy. In the relapsed OCD group, all the patients had undergone CBT (primarily based on exposure and response prevention) but had relapsed after treatment. In the treatment-naïve OCD group, 11 participants presented comorbidities on the MINI: 4 had one comorbidity, 5 presented with two comorbidities and 2 patients had three comorbidities. The most common disorders were social anxiety and agoraphobia. In the relapsed OCD group, 10 participants presented comorbidities on the MINI: 4 had one comorbidity, 2 presented with two comorbidities, 4 participants had three comorbidities. The most common disorders were social anxiety and panic disorders.

In the OCD groups, most of the patients were on treatment with a selective serotonin reuptake inhibitor (SSRI) at study intake. Three of the 19 patients in the treatment-naïve OCD group were not taking antidepressant medications, 16 were treated (SSRI). In the relapsed OCD group, except for 2 untreated patients and 2 patients taking clomipramine, all patients were on SSRI. None of the participants in the control group were receiving medical treatment.

The eligibility criteria for the treatment-naïve group and relapsed group were thus having been diagnosed as OCD patients (according to DSM-IV criteria, and

Table 1. Demographic characteristics of the study population. Data are presented as the number or the mean ( \pm standard deviation).

\begin{tabular}{cccc}
\hline & $\begin{array}{c}\text { Healthy controls } \\
(\mathrm{n}=19)\end{array}$ & $\begin{array}{c}\text { Treatment-näve OCD } \\
\text { patients }(\mathrm{n}=19)\end{array}$ & $\begin{array}{c}\text { Relapsed OCD } \\
\text { patients }(\mathrm{n}=19)\end{array}$ \\
\hline Gender ratio (males/females) & $10 / 9$ & $11 / 8$ & $12 / 7$ \\
Age (years) & $36.11(15.75)$ & $35.53(7.44)$ & $36.63(11.35)$ \\
Duration of illness (years) & - & $16.26(5.42)$ & $19.32(12.18)$ \\
Years of formal education & $15.21(3.54)$ & $14.89(3.28)$ & $13.68(2.29)$ \\
\hline
\end{tabular}


VOCI total score). The main exclusion criteria were previous or current neurological disorders (such as traumatic head injury, epilepsy, stroke, and dementia), the use of psychotropic medication, or previous or current schizophrenia. The use of alcohol and or (soft) drugs was an exclusion criterion.

The psychopathological characteristics of the study groups are summarized in Table 2. The OCD symptoms and related beliefs were rated using the Vancouver Obsessive-Compulsive Inventory (VOCI, [24]) and the 44-item Obsessive Beliefs Questionnaire (OBQ-44, [25]). The severity of depression was assessed using the Beck Depression Inventory (BDI, [26]). Higher scores in those questionnaires mean severity. All participants gave their written informed consent, and the research was approved by the institutional review board (Chambéry 2014, No. 20146) and considered as compatible with the ethical guidelines of the declaration of Helsinki.

\subsection{Material and Procedure}

\section{1) Assessment of the clinical and psychological background:}

The VOCI [24] is a 55-item self-reported measure designed to evaluate OCD symptoms on six subscales: checking, contamination, obsessions, hoarding, "just right" and indecisiveness. The OBQ-44 [25] assesses beliefs considered to be important in the development and maintenance of OCD, using three subscales: responsibility/threat estimation; perfectionism/uncertainty; and importance/control of thoughts. The 21-item BDI [26] indicates the severity of depression.

Table 2. Self-reported questionnaire data for the study groups. Data are presented as the mean ( \pm standard deviation).

\begin{tabular}{lccc}
\hline Self-reported questionnaire/subscale & $\begin{array}{c}\text { Healthy controls } \\
(\mathbf{n}=19)\end{array}$ & $\begin{array}{c}\text { Treatment-naïve } \\
\text { OCD patients } \\
(\mathbf{n}=19)\end{array}$ & $\begin{array}{c}\text { Relapsed OCD } \\
\text { patients } \\
(\mathbf{n}=19)\end{array}$ \\
\hline VOCI total score (/220) & $26.68(17.59)$ & $97.79(35.65)$ & $93.53(38.37)$ \\
Checking (/24) & $8.26(4.76)$ & $26.42(9.17)$ & $24.74(9.78)$ \\
Contamination (/48) & $5.37(5.08)$ & $25.74(12.71)$ & $18.53(12.45)$ \\
Obsessions (/48) & $3.11(3.23)$ & $7.84(8.38)$ & $7.32(7.87)$ \\
Hoarding (/28) & $4.47(4.55)$ & $15.11(10.28)$ & $18.11(11.83)$ \\
Just right (/48) & $2.58(2.43)$ & $10.89(7.59)$ & $12.89(7.49)$ \\
Indecisiveness (/24) & $3.42(2.83)$ & $11.79(5.33)$ & $11.95(5.64)$ \\
OBQ-44 total score (/308) & $114.79(38.24)$ & $186.42(48.49)$ & $172.63(52.66)$ \\
Responsibility/Threat estimation (/112) & $42.42(19.12)$ & $70.21(24.96)$ & $69.11(27.80)$ \\
Perfectionism/Uncertainty (/112) & $48.11(14.63)$ & $74.26(16.97)$ & $64.74(22.28)$ \\
Importance/Control of Thoughts (/84) & $24.26(11.68)$ & $41.95(17.00)$ & $38.79(19.34)$ \\
BDI score (/63) & $5.26(4.79)$ & $19.50(9.56)$ & $19.95(9.92)$ \\
\hline
\end{tabular}

Note: VOCI: Vancouver Obsessive Compulsive Inventory; OBQ-44: Obsessional Beliefs Questionnaire-44 items; BDI: Beck Depression Inventory. 


\section{2) Evaluation of EF:}

The 75-item BRIEF-A [27] assesses behaviours associated with EF deficits in everyday life in adults. The French version of the questionnaire [28] has been validated in adults aged from 18 to 93. Behaviours are rated in terms of their ability to inhibit impulsive responses (the "Inhibit" subscale), adjust to changes in routine or task demands ("Shift"), modulate emotions ("Emotional Control"), monitor one's own behaviour ("Self-Monitor"), initiate problem solving or activity ("Initiate"), sustain working memory ("Working Memory"), plan and organize problem solving approaches("Plan/Organize"), monitor a task ("Task Monitor") and organize environment and materials ("Organization of Materials").

Four of the clinical subscales (Inhibit, Shift, Emotional Control, and Self-Monitor) form a Behavioral Regulation Index (BRI) of the self-regulation of behaviour and emotions. Five of the subscales (Initiate, Working Memory, Plan/Organize, Task Monitor, and Organization of Materials) form a Metacognition Index (MI), which evaluates the executive regulation of cognitive problem solving. Lastly, a Global Executive Composite (GEC) score comprises all the subscales. Participants are asked to rate their behaviour in the last six months on a three-point Likert scale, according to whether the behaviour has "never" (coded as 1), "sometimes" (coded as 2), or "often" (coded as 3) been a problem. These answers are then summed for each clinical subscale and index, and transformed into age-appropriate T-scores based on normative data [28]. Higher T scores reflect greater impairments in EF.

\subsection{Analyses}

Statistical analyses were performed using Statistica software (version 10). Demographic and clinical characteristics were analyzed using a one-way between-subjects analysis of variance.

A multivariate analysis of variance was performed on the BRIEF-A results, and univariate $\mathrm{F}$ tests were used to assess intergroup effects for each subscale and each index; the threshold for statistical significance was set to $p<0.05$. Effect sizes were calculated by using the partial $\eta^{2}$ method. When a statistically significant result was detected, we applied a post hoc Tukey honest significant difference (HSD) test in order to compare each group with the other two groups.

Correlational analysis (Spearman's coefficient) was used to investigate the relationships between the BRIEF-A variables and the demographic and clinical variables; in order to take the multiple analyses into account, the threshold for statistical significance was set to a more restrictive value $(\mathrm{p}<0.01)$.

\section{Results}

\subsection{Demographic and Clinical Characteristics}

The study participants' demographic and clinical characteristics are summarized in Table 1 and Table 2. 
- There were no statistically significant differences between the three groups with regard to age $\left(\mathrm{F}(2,54)=0.04, \mathrm{p}=0.96, \eta^{2} \mathrm{p}=0.001\right)$ or education level $\left(\mathrm{F}(2,54)=1.30, \mathrm{p}=0.28, \eta^{2} \mathrm{p}=0.05\right)$. The two OCD groups did not differ significant with regard to the duration of illness $\left(\mathrm{F}(1,36)=1.00, \mathrm{p}=0.32, \eta^{2} \mathrm{p}\right.$ $=0.03$ ).

- A significant effect of group was observed for the VOCI total score $(F(2,54)$ $=29.69, \mathrm{p}<0.001, \eta^{2} \mathrm{p}=0.52$ ). Post hoc comparisons (using the Tukey HSD test) indicated that the mean scores for the treatment-naïve OCD group ( $\mathrm{M}=$ $97.79, \mathrm{SD}=35.65)$ and the relapsed OCD group $(\mathrm{M}=93.53, \mathrm{SD}=38.37)$ differed significantly $(\mathrm{p}<0.001)$ from the mean score in the healthy control group $(M=26.68, S D=17.59)$. The relapsed $O C D$ group did not differ significantly from the treatment-naive OCD group. The same effects were found for five of the six VOCI subscales: checking $\left(\mathrm{F}(2,54)=28.34, \mathrm{p}<0.001, \eta^{2} \mathrm{p}=\right.$ $0.51)$, contamination $\left(\mathrm{F}(2,54)=17.75, \mathrm{p}<0.001, \eta^{2} \mathrm{p}=0.40\right)$, hoarding $(\mathrm{F}(2$, $\left.54)=10.98, \mathrm{p}<0.001, \eta^{2} \mathrm{p}=0.29\right)$, “just right" $(\mathrm{F}(2,54)=14.25, \mathrm{p}<0.001$, $\left.\eta^{2} \mathrm{p}=0.35\right)$, and indecisiveness $\left(\mathrm{F}(2,54)=19.87, \mathrm{p}<0.001, \eta^{2} \mathrm{p}=0.42\right)$. The scores for each of the two OCD groups were significant higher than for the healthy control group-confirming the presence of OCD symptom in the patients. There was no difference between the treatment-naïve OCD group and the relapsed OCD group in a post hoc Tukey HSD test.

- For the OBQ-44, there was a significant effect of group on the total score $\left(\mathrm{F}(2,54)=8.50, \mathrm{p}<0.001, \eta^{2} \mathrm{p}=0.32\right)$ and each factor score: Responsibility/Threat estimation $\left(\mathrm{F}(2,54)=12.50, \mathrm{p}<0.001, \eta^{2} \mathrm{p}=0.23\right)$, Perfectionism/Uncertainty $\left(\mathrm{F}(2,54)=10.01, \mathrm{p}<0.001, \eta^{2} \mathrm{p}=0.27\right)$, and Importance/Control of Thoughts $\left(\mathrm{F}(2,54)=6.34, \mathrm{p}=0.003, \eta^{2} \mathrm{p}=0.19\right)$. Again, for each of the OBQ-44 measures, a post hoc Tukey HSD test showed that each patient group had significantly higher scores than the healthy control group $(\mathrm{p}<0.05)$, with no difference between the treatment-naïve OCD group and the relapsed OCD group.

- There was no statistically significant overall difference in the VOCI or OBQ-44 scale scores between the treatment-naïve OCD group and the relapsed OCD group-indicating a similar level of severity.

- According to the BDI, the depression score differed significantly from one group to another $\left(\mathrm{F}(2,54)=18.62, \mathrm{p}<0.001, \eta^{2} \mathrm{p}=0.41\right)$. The treatment-naïve OCD group $(M=19.50, S D=9.56)$ and the relapsed $\mathrm{OCD}$ group $(\mathrm{M}=19.95$, $\mathrm{SD}=9.92)$ had significantly higher scores than the HC group $(\mathrm{p}<0.001)$, with no difference between the two patient groups.

\subsection{Behavioural EF in Everyday Life: BRIEF-A Results}

Table 3 summarizes the proportion of participants in each of the study groups with abnormal EF T-scores (i.e. >65) for each BRIEF-A subscale and index.

- The proportion of participants with abnormal EF T-scores was higher in the treatment-naïv OCD group and the relapsed OCD group than in the healthy control group. We performed a multivariate analysis of variance with the 
BRIEF-A T-scores (the nine clinical scales and the three indices-BRI, MI and the GEC score as a measure of overall adjustment) as dependent variables and group as the independent variable (Wilks $=0.25 ; \mathrm{F}(12,24)=3.60$, $\mathrm{p}$ $<0.001)$.

- According to the BDI, patients with OCD were more depressed than healthy controls. Given that depression might have influenced the dependent variables (i.e. the EF scores, [29]), the BDI total score was used as a covariate in the following analyses.

- The follow-up univariate F tests were statistically significant for seven of the 12 factor scores, with a trend towards a significant group effect for the MI. T-scores for Inhibit, Working Memory, Task Monitor and Organization of Material did not achieve statistical significance. Table 4 presents the mean T-scores for each BRIEF-A subscale and index in each group, and the main intergroup differences.

Table 3. Percentage of participants in the three study groups with impairments in the BRIEF-A subscales and indices.

\begin{tabular}{cccc}
\hline \multirow{2}{*}{ BRIEF-A subscale or index } & \multicolumn{3}{c}{ Study group } \\
\cline { 2 - 4 } & $\begin{array}{c}\text { Healthy controls } \\
(\mathrm{n}=19)\end{array}$ & $\begin{array}{c}\text { Treatment-naïv OCD } \\
\text { patients }(\mathrm{n}=19)\end{array}$ & $\begin{array}{c}\text { Relapsed OCD } \\
\text { patients (n = 19) }\end{array}$ \\
\hline Inhibit & $16 \%$ & $32 \%$ & $26 \%$ \\
Shift & $0 \%$ & $63 \%$ & $63 \%$ \\
Emotional Control & $11 \%$ & $58 \%$ & $16 \%$ \\
Self-Monitor & $0 \%$ & $37 \%$ & $5 \%$ \\
Behavior Regulation Index & $0 \%$ & $63 \%$ & $26 \%$ \\
Initiate & $11 \%$ & $63 \%$ & $74 \%$ \\
Working Memory & $11 \%$ & $63 \%$ & $58 \%$ \\
Plan/Organize & $11 \%$ & $58 \%$ & $37 \%$ \\
Task Monitor & $0 \%$ & $37 \%$ & $21 \%$ \\
Organization of Materials & $0 \%$ & $26 \%$ & $11 \%$ \\
Metacognition Index & $11 \%$ & $47 \%$ & $47 \%$ \\
Global Executive Composite & $5 \%$ & $63 \%$ & $42 \%$ \\
\hline
\end{tabular}

Table 4. Mean ( \pm standard deviation) T-scores for the BRIEF-A subscales and indices in the three study groups.

\begin{tabular}{|c|c|c|c|c|}
\hline \multirow{4}{*}{ BRIEF-A subscale or index } & \multicolumn{3}{|c|}{ Study group } & \multirow{4}{*}{ Group differences } \\
\hline & \multirow{3}{*}{$\begin{array}{c}\begin{array}{c}\text { Healthy } \\
\text { controls }\end{array} \\
\text { Mean (SD) }\end{array}$} & \multicolumn{2}{|c|}{ Treatment-naïveRelapsed OCD } & \\
\hline & & diterits & patients & \\
\hline & & Mean (SD) & Mean (SD) & \\
\hline Inhibit & $50.00(9.22)$ & $59.32(12.66)$ & $53.32(14.29)$ & $\mathrm{C}<\mathrm{TN}-\mathrm{OCD}^{*}$ \\
\hline Shift & $49.74(10.69)$ & $70.95(11.26)$ & $70.32(11.94)$ & $\mathrm{C}<\mathrm{TN}-\mathrm{OCD}, \mathrm{R}-\mathrm{OCD}$ \\
\hline Emotional Control & $51.68(10.31)$ & $65.53(8.66)$ & $55.84(12.07)$ & C, $\mathrm{R}-\mathrm{OCD}<\mathrm{TN}-\mathrm{OCD}$ \\
\hline
\end{tabular}




\section{Continued}

\begin{tabular}{|c|c|c|c|c|}
\hline Self-Monitor & $47.84(7.32)$ & $57.32(12.84)$ & $47.37(8.76)$ & C, R-OCD $<$ TN-OCD \\
\hline Behavior Regulation Index & 50.26 & $66.79(10.02)$ & $57.89(12.07)$ & $\begin{array}{c}\text { C }<\text { TN-OCD, R-OCD; } \\
\text { R-OCD }<\text { TN-OCD }\end{array}$ \\
\hline Initiate & $50.42(8.68)$ & $72.47(14.65)$ & $73.26(13.85)$ & $\mathrm{C}<\mathrm{TN}-\mathrm{OCD}, \mathrm{R}-\mathrm{OCD}$ \\
\hline Working Memory & $50.53(8.47)$ & $68.21(14.65)$ & $63.32(15.67)$ & $\mathrm{C}<\mathrm{TN}-\mathrm{OCD}, \mathrm{R}-\mathrm{OCD}$ \\
\hline Plan/Organize & $48.05(9.83)$ & $65.58(14.79)$ & $63.68(11.85)$ & $\mathrm{C}<\mathrm{TN}-\mathrm{OCD}, \mathrm{R}-\mathrm{OCD}$ \\
\hline Task Monitor & $49.74(8.61)$ & $59.32(15.96)$ & $55.37(14.06)$ & $\mathrm{C}<\mathrm{TN}-\mathrm{OCD}^{*}$ \\
\hline Organization of Materials & $48.89(9.51)$ & $52.58(11.36)$ & $51.63(11.84)$ & $\mathrm{C}=\mathrm{TN}-\mathrm{OCD}=\mathrm{R}-\mathrm{OCD}$ \\
\hline Metacognition Index & $49.26(9.90)$ & $67.11(14.84)$ & $66.79(13.52)$ & $\mathrm{C}<\mathrm{OCD}, \mathrm{R}-\mathrm{OCD}$ \\
\hline Global Executive Composite & $49.68(9.73)$ & $68.58(12.19)$ & $62.26(12.79)$ & $\mathrm{C}<\mathrm{TN}-\mathrm{OCD}, \mathrm{R}-\mathrm{OCD}$ \\
\hline
\end{tabular}

SD: standard deviation; TN-OCD: obsessive compulsive disorder; R-OCD: obsessive compulsive disorder relapse; $\mathrm{C}$ : healthy control participants; *: non-significant trend.

- There was a significant effect of group on the GEC T-score $(F(2,52)=4.63$, $\mathrm{p}$ $\left.=0.01, \eta^{2} \mathrm{p}=0.15\right)$. The difference between the treatment-naïve OCD group and the relapsed OCD group was significant $(\mathrm{p}<0.01)$, as was the difference between the treatment-naïve OCD group and the healthy control group $(\mathrm{p}<$ 0.01). However, in a post hoc Tukey HSD test, the relapsed OCD group did not differ from the healthy control group in terms of the GEC score. A significant difference on the BRI T-scores was observed between the 3 groups $\left[\mathrm{F}(2,52)=5.36, \mathrm{p}<0.01, \eta^{2} \mathrm{p}=0.17\right]$. A post hoc Tukey HSD test showed a significant difference between the treatment-naïve OCD group and the healthy controls $(\mathrm{p}<0.01)$. The treatment-naïve OCD group had significantly greater $\mathrm{T}$-scores than the relapsed OCD group $(\mathrm{p}=0.02)$. For the MI score, there was a non-significant trend towards a group effect $[\mathrm{F}(2,52)=$ 2.69, $\mathrm{p}=0.08, \eta^{2} \mathrm{p}=0.09$ ], with significant differences between the treatment-naïve OCD group and the healthy control group $(\mathrm{p}<0.01)$, between the relapsed OCD group and the healthy control group $(\mathrm{p}<0.01)$, but not between the treatment-naïve $\mathrm{OCD}$ group and relapsed $\mathrm{OCD}$ group $(\mathrm{p}=$ 0.90).

An analysis of the BRIEF-A subscale scores revealed:

- A distinct trend towards a significant difference in the Inhibit subscale $[\mathrm{F}(2$, $\left.52)=1.91, \mathrm{p}=0.16, \eta^{2} \mathrm{p}=0.07\right]$; the results of a posthoc Tukey HSD tests indicated a significant difference between treatment-naïve OCD group and the healthy controls $(\mathrm{p}<0.05)$ but no difference between the treatment-naïve OCD group and the relapsed OCD group $(p=0.25)$ or between the relapsed OCD group and healthy controls $(\mathrm{p}=0.69)$.

- A significant effect of group on the Shift T-scores $[F(2,52)=6.90, p<0.01$, $\left.\eta^{2} \mathrm{p}=0.21\right]$. Post hoc Tukey HSD tests indicated that the treatment-naïve OCD group and the relapsed OCD group differed significantly from control participants $(\mathrm{p}<0.01)$, with no difference between the two groups of OCD patients $(\mathrm{p}=0.93)$. 
- Significant effects of group on the Emotional Control T-scores $[\mathrm{F}(2,52)=$ 4.53, $\left.\mathrm{p}=0.02, \eta^{2} \mathrm{p}=0.15\right]$ and the Self Monitor T-scores $[\mathrm{F}(2,52)=5.26, \mathrm{p}<$ $0.01, \eta^{2} \mathrm{p}=0.17$, with a significant difference between the treatment-naïve OCD group and the healthy control group $(\mathrm{p} \leq 0.01)$, between the treatment-naïve OCD group and the relapsed OCD group ( $<<0.05)$, but not between the relapsed OCD group and the healthy control group.

- A significant effect of group on the Initiate $\mathrm{T}$-scores $[\mathrm{F}(2,52)=7.93, \mathrm{p}<$ $\left.0.01, \eta^{2} \mathrm{p}=0.23\right]$. Tukey pairwise comparisons were statistically significant $(\mathrm{p}<0.01)$ between the treatment-naïve OCD group and the relapsed OCD group, between the treatment-naïve OCD group and healthy controls, but not between the treatment-naïve OCD group and the relapsed OCD group $(\mathrm{p}=0.96)$.

- No significant group effect on the Working Memory T-score $[F(2,52)=2.21$, $\left.\mathrm{p}=0.12, \eta^{2} \mathrm{p}=0.08\right]$, although Tukey pairwise comparisons showed significant differences between the two patient groups and the healthy control group ( $\mathrm{p}<0.01$ ) but no difference between the two OCD groups $(\mathrm{p}=0.30$ ).

- A significant effect of group on the Plan/Organize T-scores $[F(2,52)=4.46$, $\left.\mathrm{p}=0.02, \eta^{2} \mathrm{p}=0.15\right]$. The Tukey comparisons showed a statistically significant difference $(\mathrm{p}<0.01)$ between the treatment-naïve OCD group and the relapsed OCD group, between the treatment-naïve OCD group and healthy controls, but not between the treatment-naïv OCD group and the relapsed $\operatorname{OCD} \operatorname{group}(\mathrm{p}=0.61)$.

- No significant differences for the Task Monitor $\left[\mathrm{F}(2,52)=0.57, \mathrm{p}=0.57, \eta^{2} \mathrm{p}\right.$ $=0.02]$ and Organization of Materials $\left[\mathrm{F}(2,52)=0.12, \mathrm{p}=0.88, \eta^{2} \mathrm{p}=0.004\right]$ $\mathrm{T}$-scores. The Tukey pairwise comparisons revealed a non-significant trend towards a difference between the treatment-naïve OCD group and the healthy controls in the Task Monitor score $(\mathrm{p}=0.06)$.

\subsection{Correlations between Clinical Data and Executive Scores in Patients with OCD}

Spearman correlations were examined between clinical data and BRIEF-A indices, with a $\mathrm{p}$ value fixed at 0.01 after Bonferroni correction for multiple comparisons.

- The severity of illness (according to the VOCI total score) was significantly and positively correlated with the BRIEF-A index scores: the BRI $(\mathrm{r}=0.42, \mathrm{p}$ $<0.01)$, MI $(\mathrm{r}=0.47, \mathrm{p}<0.01)$ and GEC score $(\mathrm{r}=0.51, \mathrm{p}<0.01)$.

- Obsessive beliefs were significantly and positively correlated with two BRIEF-A indices: the BRI $(r=0.47, \mathrm{p}<0.01)$ and GEC score $(\mathrm{r}=0.45, \mathrm{p}<$ $0.01)$.

- The duration of illness was not correlated with the severity of OCD as measured by the VOCI $(r=0.14, p>0.01)$, or OBQ-44 $(r=0.06, p>0.01)$, total scores, nor with any of the BRIEF-A variables: BRI $(r=0.06, p>0.01)$, MI ( $r$ $=0.20, \mathrm{p}>0.01)$ and GEC $(\mathrm{r}=0.11, \mathrm{p}>0.01)$ 


\section{Discussion}

With regard to demographic variables, the three study groups were matched for gender, age and educational level. As expected, patients in the treatment-naive OCD group and the relapsed OCD group had significantly higher scores than the control group participants for all questionnaire scales and indices (VOCI, $\mathrm{OBQ}$ and $\mathrm{BDI}$ ), with the exception of the VOCI obsession subscale. According to the VOCI, two types of intrusive thoughts (checking and contamination) were prevalent in both two groups of patients. The clinical symptom profile, OCD severity, medication and duration of illness were similar in the two groups of patients.

One of our hypothesis was that higher impairments in EF were predictive of higher obsessive -compulsive severity. Our analyses revealed positive significant correlations between the measures of OCD severity (VOCI and OBQ-44) and BRIEF-A main scores: the more severe the OCD symptoms, the more the BRIEF-A scores reflected an impairment in EF. This result is consistent with McNamara et al's study [16], who found in children with OCD that impairments in various domains of EF were predictive of higher mean obsessive compulsive severity scores. In adults with OCD, Pedron et al. [30] showed that specific impairment of executive functions was associated with specific obsessive-compulsive symptoms dimensions (e.g. symmetry/ordering, hoarding, contamination/cleaning...), the severity of obsessive-compulsive symptoms in those different dimensions significantly correlating with impaired specific executive functions. This association between EF impairment and symptoms severity could be explained by the frontosubcortical circuitry deficits in OCD, influencing both clinical symptoms and executive dysfunctions, creating a vicious circle [31].

Our correlation results also showed that the duration of illness was not related to OCD symptom severity (VOCI and OBQ-44 scores) or to EF impairment (BRIEF-A scores).

A further question is whether there is a global or specific impairment in EF in OCD. Our first starting hypothesis was that treatment-naive OCD patients and patients having relapsed after CBT (based on exposure and response prevention) would display impaired EF in everyday life (as assessed by the BRIEF-A).

Our results showed that $63 \%$ of the patients in the treatment-naive OCD group and $42 \%$ of the patients in the relapsed OCD group had an overall deficit in the GEC score, compared with just $5 \%$ of the healthy controls, with more difficulties in behavioural regulation: for the BRI, $63 \%$ of the treatment-naïve patients had clinically significant deficits versus $26 \%$ of the relapsed OCD patients, none of the control participants being impaired. Analyses on the T-scores in the BRIEF-A showed that the GEC score was significantly impaired in both groups of patients, relative to matched control participants, with no significant difference between the two OCD groups, indicating global impairment of all EF domains both in treatment-naive and relapsed OCD patients. Significant differences for treatment-naïve OCD patients and relapsed OCD patients (relative to 
healthy controls) were found for the BRI and the MI. These results are in line with the impairment of EF in OCD observed in many [9].

In most of the sub-scales (shift, initiate, working memory, plan/organize, task monitor) post-hoc analyses revealed significant EF deficits in the two OCD groups relatively to healthy control participants. This pattern of results in the Initiate subscale is not surprising because compulsions lead to delay in performing an action or initiating an activity in many situations.

However, in contrast to our hypothesis, inhibitory processes (according to the BRIEF-A) did not seem to be particularly impaired in either of the two OCD groups; there was a non-significant trend in the treatment-naïve OCD group and no difference in the relapsed OCD group, relative to healthy controls. As a sub-component of cognitive control, inhibition is usually considered to have two main components: cognitive inhibition (the ability to tune out the stimuli irrelevant to the task/process at hand, in order to process the relevant stimuli [32], [33] and behavioural inhibition (control over motor activities). Chamberlain et al. [1] suggested that the failures in cognitive inhibitory processes in OCD are due to a lack of control over internal cognition (e.g. intrusive thoughts) and over the behavioural inhibition of compulsions (mostly ritualistic checking behaviour). Although the BRIEF-A examines behavioural inhibition (rather than cognitive inhibition), impairments in inhibition appear to be more specifically linked to a tendency to act on impulse, whereas the impulsion in OCD may be more specifically linked to compulsivity (i.e. a tendency to repeat certain behaviours). The subdivision in behavioural inhibition between impulsivity and compulsivity [34] might explain the lack of an impairment for the BRIEF-A Inhibit subscale in the two patient groups. Another point needs to be emphasized: $16 \%$ of the healthy control participants, $26 \%$ of the relapsed OCD patients and $32 \%$ of the treatment-naïv OCD patients had a significant deficit in this subscale (e.g. T-scores $>65$ ). The percentages of OCD patients with a deficit in the Inhibition subscale were not particularly high, and the percentage of control participants was surprisingly high; this may have contributed to the lack of a significant difference between patients and control participants for this subscale.

As a conclusion, the EF impairment in everyday life seems more general than specific in OCD as most of the sub-scales were impaired in the BRIEF-A.

Another question in our experiment was whether there is a difference in the degree of impairment between treatment-naïve OCD patients and relapsed OCD patients. As seen before, in most of the sub-scales, post-hoc analyses revealed significant EF deficits in the two OCD groups relatively to healthy control participants, with the same level of impairment in the two groups of patients. Surprisingly, the relapsed OCD group had lower scores for the BRI (i.e. better behavioural regulation skills) than the treatment-naïve OCD group. Given that the CBT undertaken by the relapsed OCD group was mainly based on exposure and response prevention, one can hypothesize that the group's better behavioural regulation skills were due to a treatment effect. No difference between the two 
OCD groups was observed for the MI.

For the Emotional Control and Self Monitor scales, the treatment-naïve OCD group was significantly impaired relatively to healthy controls, but not the relapsed OCD group. As for the BRI, a positive effect of the CBT in the relapsed group could be inferred as the severity of symptoms, duration of illness and pharmacological treatments were quite the same in the two OCD groups. One could infer that the CBT had an impact on emotional processes that influence complex behavior in the "real world".

Overall, the relapsed OCD group does not exhibit a greater impairment in EF in everyday life than the treatment-naïve OCD group, regardless of the subscale (Inhibit, Shift, Emotional Control, Self Monitor, Initiate, Working Memory, Plan/Organize, Task Monitor, Organization of Materials). In two sub-scales (Inhibit and Task Monitor), lesser T-scores were observed in the relapsed OCD group relatively to the treatment-naïve OCD group, showing a better EF. The relapsed OCD group had a better BRI than the treatment-naïve OCD group, whereas the two groups of patients had similar scores for the MI and the GEC score.

Finally, the last question is about whether EF performance in OCD is state or trait-dependent. Bannon et al.'s results [10] suggest that 1 ) not all aspects of EF are impaired in OCD individuals, and 2) EF impairments and non-impairments remain stable over the course of the disorder and do not deteriorate with the duration of the illness or improve with symptom remittance. In the present study, no significant correlations were found between EF performance and the duration of illness.

The absence of difference in EF between the two OCD groups could inform us about whether executive abilities are trait or state related. Our results suggest that EF impairments are more state-dependent than trait-dependent. The same level of obsessive-compulsive symptoms after relapse from CBT (relapsed OCD group) than before treatment (treatment-naïve group) could be linked to a return to the same level of EF impairment in the relapsed group. However, to ensure this hypothesis it would be better to compare a same group of OCD patients before CBT and after having relapsed from therapy. Indeed, our study used a cross-sectional design. This may have significant shortcomings, since the patients' trajectories (i.e. developmental, family and clinical histories) make it difficult to match individuals across groups. Furthermore, the naïve group could contain potential patients who could relapse after having followed CBT.

The study also had some limitations: 1) a relatively small sample size, 2) the lack of a recovered post-treatment group 3) the lack of a control group with another disorder to determine the extent to which executive deficits are specific to OCD or general impairment relative to a clinical condition 4) a lack of data on the reasons why CBT failed in the relapsed OCD group, 5) the BRIEF-A only evaluates the repercussions of impaired EF on everyday life, rather than EF per se (i.e. in laboratory tests), 6) informant report of the BRIEF-A was not admin- 
istered and could also provide meaningful information on the patient's functioning in everyday life.

\section{Conclusions}

Treatment-naïve or relapsed OCD patients reported poor EF in everyday life, according to the BRIEF-A questionnaire. Relative to control participants, behavioural regulation and metacognition were especially impaired. The two OCD groups were equally impaired in terms of shifting, initiating and working memory behaviours than the control group. Hence, the relapsed OCD group did not report a greater impairment in behavioural inhibition or in the other dimensions of EF measured by the BRIEF-A than the treatment naïve group, which suggest that EF impairments are more state-dependent.

One of the present study's strengths relates to its investigation of several domains of EF in everyday life in two groups of patients with OCD (treatment-naïve patients and post-CBT relapsed patients) and healthy control participants. The BRIEF-A questionnaire is relatively easy to administer because it does not require neuropsychological testing and allows a better knowledge about a patient's executive deficits and their relationships to OCD symptoms. Evaluating the executive functioning of a patient by means of the BRIEF-A would allow the clinician to check whether the patient with OCD is not too disabled in everyday life, which could affect the care. It could be necessary then, to begin therapeutic interventions with targeted cognitive remediation of executive functioning, which may not only help OCD patients with their executive dysfunction but perhaps also ameliorate their obsessive-compulsive symptomatology.

\section{Acknowledgements}

We thank David Fraser (Biotech Communication, Damery, France) for helpful comments on the manuscript's English.

\section{Conflicts of Interest}

The authors declare no conflicts of interest regarding the publication of this paper.

\section{References}

[1] Chamberlain, S.R., Blackwell, A.D., Fineberg, N.A., Robbins, T.W. and Sahakian, B.J. (2005) The Neuropsychology of Obsessive Compulsive Disorder: The Importance of Failures in Cognitive and Behavioural Inhibition as Candidate Endophenotypic Markers. Neuroscience and Biobehavioral Reviews, 29, 399-419. https://doi.org/10.1016/j.neubiorev.2004.11.006

[2] Miyake, A., Friedman, N.P., Emerson, M.J., Witzki, A.H., Howerter, A. and Wager, T.D. (2000) The Unity and Diversity of Executive Functions and Their Contributions to Complex Frontal Lobe Tasks: A Latent Variable Analysis. Cognitive Psychology, 41, 49-100. https://doi.org/10.1006/cogp.1999.0734

[3] Diamond, A. (2013) Executive Functions. Annual Review of Psychology, 64, 135-168. https://doi.org/10.1146/annurev-psych-113011-143750 
[4] Alexander, G.E. and Crutcher, M.D. (1990) Functional Architecture of Basal Ganglia Circuits: Neural Substrates of Parallel Processing. Trends in Neurosciences, 13, 266-271. https://doi.org/10.1016/0166-2236(90)90107-L

[5] Cummings, J.L. (1993) Frontal-Subcortical Circuits and Human Behavior. Archives of Neurology, 50, 873-880. https://doi.org/10.1001/archneur.1993.00540080076020

[6] Chamberlain, S.R., Menzies, L., Hampshire, A., Suckling, J., Fineberg, N.A., del Campo, N., Aitken, M., Craig, K., Owen, A.M., Bullmore, E.T., Robbins, T.W. and Sahakian, B.J. (2008) Orbitofrontal Dysfunction in Patients with Obsessive-Compulsive Disorder and Their Unaffected Relatives. Science, 321, 421-422. https://doi.org/10.1126/science.1154433

[7] Maia, T.V., Cooney, R.E. and Peterson, B.S. (2008) The Neural Bases of Obsessive-Compulsive Disorder in Children and Adults. Development and Psychopathology, 20, 1251-1283. https://doi.org/10.1017/S0954579408000606

[8] Nakao, T., Okada, K. and Kanba, S. (2014) Neurobiological Model of Obsessive-Compulsive Disorder: Evidence from Recent Neuropsychological and Neuroimaging Findings. Psychiatry and Clinical Neurosciences, 68, 587-605.

https://doi.org/10.1111/pcn.12195

[9] Snyder, H., Kaiser, R., Warren, S. and Heller, W. (2015) Obsessive-Compulsive Disorder Is Associated with Broad Impairments in Executive Function: A Meta-Analysis. Clinical Psychological Science, 3, 301-330. https://doi.org/10.1177/2167702614534210

[10] Bannon, S., Gonsalvez, C.J., Croft, R.J. and Boyce, P.M. (2006) Executive Functions in Obsessive-Compulsive Disorder: State or Trait Deficits? Australian and New Zealand Journal of Psychiatry, 40, 1031-1038.

[11] Olley, A., Malhi, G. and Sachdev, P. (2007) Memory and Executive Functioning in Obsessive-Compulsive Disorder: A Selective Review. Journal of Affective Disorders, 104, 15-23. https://doi.org/10.1016/j.jad.2007.02.023

[12] Markarian, Y., Larson, M., Aldea, M., Baldwin, S., Good, D., Berkeljon, A., Murphy, T.K., Storch, E.A. and McKay, D. (2010) Multiple Pathways to Functional Impairment in Obsessive-Compulsive Disorder. Clinical Psychology Review, 30, 78-88. https://doi.org/10.1016/j.cpr.2009.09.005

[13] Kueltz, A.K., Hohagen, F. and Voderholzer, U. (2004) Neuropsychological Performance in Obsessive-Compulsive Disorder: A Critical Review. Biological Psychology, 65, 185-236. https://doi.org/10.1016/j.biopsycho.2003.07.007

[14] Abramowitch, A., Abramowitz, J.S. and Mittelman, A. (2013) The Neuropsychology of Adult Obsessive-Compulsive Disorder: A Meta-Analysis. Clinical Psychology Review, 33, 1163-1171. https://doi.org/10.1016/j.cpr.2013.09.004

[15] Burgess, P.W., Alderman, N., Evans, J., Emslie, H. and Wilson, B.A. (1998) The Ecological Validity of Tests of Executive Function. Journal of the International Neuropsychological Society, 4, 547-558. https://doi.org/10.1017/S1355617798466037

[16] McNamara, J., Reid, A., Balkhi, A., Bussing, R., Storch, E.A., Murphy, T.K., Graziano, P.A., Guzick, A.G. and Geffken, G.R. (2014) Self-Regulation and Other Executive Functions Relationship to Paediatric OCD Severity and Treatment Outcome. Journal of Psychopathology and Behavioral Assessment, 36, 432-442. https://doi.org/10.1007/s10862-014-9408-3

[17] Chaytor, N.S., Schmitter-Edgecombe, M. and Burr, R. (2006) Improving the Ecological Validity of Executive Functioning Tests: Environmental Demands and Compensatory Strategies. Archives of Clinical Neuropsychology, 21, 217-227. 
https://doi.org/10.1016/j.acn.2005.12.002

[18] Abramovitch, A. and Cooperman, A. (2015) The Cognitive Neuropsychology of Obsessive-Compulsive Disorder: A Critical Review. Journal of Obsessive-Compulsive and Related Disorders, 5, 24-36. https://doi.org/10.1016/j.jocrd.2015.01.002

[19] Baioui, A., Pilgramm, J., Kagerer, S., Walter, B., Vaitl, D. and Stark, R. (2013) Neural Correlates of Symptom Reduction after CBT in Obsessive-Compulsive Washers-An fMRI Symptom Provocation Study. Journal of Obsessive-Compulsive and Related Disorders, 2, 322-330. https://doi.org/10.1016/j.jocrd.2013.04.006

[20] Voderholzer, U., Schwartz, C., Freyer, T., Zurowski, B., Thiel, N., Herbst, N., Wahl, K., Kordon, A., Hohagen, F. and Kuelz, A.K. (2013) Cognitive Functioning in Medication-Free Obsessive-Compulsive Patients Treated with Cognitive-Behavioural Therapy. Journal of Obsessive-Compulsive and Related Disorders, 2, 241-248. https://doi.org/10.1016/j.jocrd.2013.03.003

[21] D’Alcante, C.C., Diniz, J.B., Fossaluza, V., Batistuzzo, M.C., Lopes, A.C., Shavitt, R.G., Deckersbach, T., Malloy-Diniz, L., Miguel, E.C. and Hoexter, M.Q. (2012) Neuropsychological Predictors of Response to Randomized Treatment in Obsessive-Compulsive Disorder. Progress in Neuro-Psychopharmacology \& Biological Psychiatry, 39, 310-317. https://doi.org/10.1016/j.pnpbp.2012.07.002

[22] Sheehan, D.V., Lecrubier, Y., Harnett Sheehan, K., et al. (1997) The Validity of the Mini International Neuropsychiatric Interview (MINI) According to the SCID-P and Its Reliability. European Psychiatry, 12, 232-241. https://doi.org/10.1016/S0924-9338(97)83297-X

[23] American Psychiatric Association (2013) Diagnostic and Statistical Manual of Mental Disorders. Fifth Edition, American Psychiatric Association, Washington DC. https://doi.org/10.1176/appi.books.9780890425596

[24] Thordarson, D.S., Radomsky, A.S., Rachman, S., Shafran, R., Sawchuk, C.N. and Hakstian, A.R. (2004) The Vancouver Obsessional Compulsive Inventory (VOCI). Behaviour Research \& Therapy, 42, 1289-1314. https://doi.org/10.1016/j.brat.2003.08.007

[25] Obsessive Compulsive Cognition Working Group (2001) Development and Initial Validation of the Obsessive Beliefs Questionnaire and the Interpretation of Intrusions Inventory. Behaviour Research and Therapy, 39, 987-1006. https://doi.org/10.1016/S0005-7967(00)00085-1

[26] Beck, A.T., Ward, C.H., Mendelson, M., Mock, J. and Erbaugh, J. (1961) An Inventory for Measuring Depression. Archives of General Psychiatry, 4, 561-571. https://doi.org/10.1001/archpsyc.1961.01710120031004

[27] Roth, R.M., Isquith, P.K. and Gioia, G.A. (2005) Behavioral Rating Inventory of Executive Function-Adult Version. Psychological Assessment Resources Inc., Lutz.

[28] Roy, A., Besnard, J., Fournet, N., Lancelot, C. and Le Gall, D. (2015) Brief-A Inventaire d'Evaluation Comportementale des Fonctions Exécutives-Version Adulte-Adaptation Française.Hogrèfe France Editions, Paris.

[29] Basso, M.R., Bornstein, R.A., Carona, F. and Morton, R. (2001) Depression Accounts for Executive Function Deficits in Obsessive Compulsive Disorder. Neuropsychiatry, Neuropsychology, and Behavioral Neurology, 14, 241-245.

[30] Pedron, A.C., Ferrão, Y.A., Gurgel, L.G. and Reppold, C.T. (2015) Relations between Executive Functions and Different Symptomatic Dimensions in Obsessive Compulsive Disorder. Paidéia (Ribeirão Preto), 25, 229-239. https://doi.org/10.1590/1982-43272561201511 
[31] Penades, R., Catalan, R., Andres, S., Salamero, M. and Gasto, C. (2005) Executive Function and Nonverbal Memory in Obsessive-Compulsive Disorder. Psychiatry Research, 133, 81-90. https://doi.org/10.1016/j.psychres.2004.09.005

[32] Nigg, J.T. (2000) On Inhibition/Disinhibition in Developmental Psychopathology: Views from Cognitive and Personality Psychology and a Working Inhibition Taxonomy. Psychological Bulletin, 126, 220-246. https://doi.org/10.1037/0033-2909.126.2.220

[33] Friedman, N.P. and Miyake, A. (2004) The Relations among Inhibition and Interference Control Functions: A Latent-Variable Analysis. Journal of Experimental Psychology: General, 133, 101-135.

https://doi.org/10.1037/0096-3445.133.1.101

[34] Bari, A. and Robbins, T.W. (2013) Inhibition and Impulsivity: Behavioral and Neural Basis of Response Control. Progress in Neurobiology, 108, 44-79.

https://doi.org/10.1016/j.pneurobio.2013.06.005 